\title{
Avaliação oftalmológica em pacientes submetidos a implante de drenagem em glaucomas refratários
}

\author{
Ophthalmologic evaluation of patients submitted to drainage implant in \\ refractory glaucoma
}

\author{
Natália Pimentel Moreno ${ }^{1}$ \\ Luciene Barbosa de Sousa ${ }^{2}$ \\ Fernanda Bon Duarte ${ }^{3}$ \\ João Guilherme Tessarioli ${ }^{4}$
}

\begin{tabular}{|c|}
\hline RESUMO \\
\hline $\begin{array}{l}\text { Objetivo: Avaliar aspectos pré e pós-operatórios em pacientes submeti- } \\
\text { dos a implantes de drenagem. Métodos: Foi realizado estudo retrospec- } \\
\text { tivo, com pacientes submetidos a implante de drenagem no setor de } \\
\text { glaucoma do Hospital Oftalmológico de Sorocaba no período de janeiro } \\
\text { de } 2004 \text { a dezembro de } 2006 \text {. No estudo foram avaliadas as seguintes } \\
\text { características: sexo, idade, olho operado, tipo de glaucoma, número de } \\
\text { cirurgias oculares anteriores, pressão intraocular prévia e após, conforme } \\
\text { tempo de seguimento. Foram observadas a acuidade visual pré e pós- } \\
\text { implante, o número de medicações antiglaucomatosas prescritas pós- } \\
\text { implante, as principais complicações e reintervenções necessárias. Resul- } \\
\text { tados: Entre os } 92 \text { pacientes a proporção foi de } 60 \text { ( } 65,3 \%) \text { do sexo } \\
\text { masculino e } 32(34,7 \%) \text { do feminino. Os principais tipos de glaucoma } \\
\text { foram: } 38,9 \% \text { (n=37) secundário a transplante de córnea e } 25,3 \% \text { (n=24) } \\
\text { glaucoma neovascular. A média da pressão intraocular pré-implante foi } \\
\text { de } 32( \pm 11,29) \text { mmHgepós-implantefoi de } 18,( \pm 8,16) \text { mmHgem } 95(100 \%) \\
\text { pacientes, } 15,00 \text { ( } \pm 11,00) \text { mmHg em } 95(100 \%) \text {, } 16 \text { ( } \pm 11,64) \text { mmHg em } 54 \\
(55,7 \%) \text { e } 20 \text { ( } \pm 10,14) \text { mmHg em } 21(21,6 \%) \text { para o período de } 2 \text { meses, } \\
6 \text { meses, } 1 \text { ano e } 2 \text { anos, respectivamente. As complicações mais frequen- } \\
\text { tes foram extrusão do implante }(8,5 \%) \text { e atalamia }(5,4 \%) \text { Conclusão: } \\
\text { Observou-se evolução satisfatória nos pós-implantes, independente das } \\
\text { diversas causas do glaucoma. Neste estudo foi encontrada redução } \\
\text { significante da pressão intraocular } 2 \text { meses, } 1 \text { ano e } 2 \text { anos (p<0,05). As } \\
\text { complicações tiveram ocorrência e evolução independente do tipo de } \\
\text { glaucoma, número de cirurgias prévias, idade e sexo. }\end{array}$ \\
\hline
\end{tabular}

Descritores: Glaucoma/cirurgia; Implantes para drenagem de glaucoma; Pressão intraocular; Complicações intraoperatórias; Acuidade visual

\section{INTRODUÇ̃̃O}

Glaucoma é uma neuropatia óptica que se apresenta na maioria dos casos de forma crônica e incurável, porém controlável. Única forma de tratamento comprovadamente eficaz até o momento é a redução da pressão intraocular. De forma geral, quando a redução não é possível com medicação, indica-se cirurgia antiglaucomatosa ${ }^{(1,2)}$. A trabeculectomia tem sido a primeira escolha entre os procedimentos cirúrgicos para tratamento do glaucoma primário de ângulo aberto, principalmente pelo alto índice de sucesso. Entretanto, isso não se aplica aos glaucomas refratários, sendo necessário o uso de dispositivos artificiais de drenagem do humor aquoso, denominados implantes de drenagem ${ }^{(3,4)}$. 
Implantes de drenagem são dispositivos artificiais que criam uma comunicação entre câmara anterior e espaço subtenoniano. Esses dispositivos consistem em um tubo ligado a um prato episcleral posterior. Alguns possuem válvulas sensíveis à pressão para regulagem do fluxo de humor aquoso $^{(5)}$, como Krupin, Ahmed e Joseph e outros não, como Molteno, Baerveldt, Susanna e Schoket ${ }^{(3,6)}$. Todos os implantes destinam-se a realizar uma bolha filtrante na região orbitária, que ocorre após a formação de uma cápsula ao redor do prato. Esta cápsula é distendida pela ação da pressão intraocular, evitando a ocorrência de uma hipotensão ocular grave. A drenagem do humor aquoso é realizada por difusão passiva através da cápsula e posteriormente pelos vasos sanguíneos adjacentes $^{(2,7)}$.

Apesar das diferenças existentes no aspecto externo dos implantes, o denominador comum é um tubo de silicone flexível, com objetivo de realizar uma fístula translimbal, conectado a explante equatorial o qual é constituído de materiais como polimetilmetacrilato, polipropileno ou silicone ${ }^{(3,6)}$.

A utilização dos implantes de drenagem para tratamento de glaucomas refratários já está consagrada pelos especialistas em glaucoma ${ }^{(3,8)}$.

As principais indicações são: glaucoma descontrolado apesar de trabeculectomia prévia com uso de antimetabólicos; glaucomas secundários em que a trabeculectomia de rotina com ou sem uso de antimetabólicos seria provavelmente ineficaz (glaucoma neovascular, secundários a traumas graves e aniridia); grave cicatriz conjuntival que impede uma boa dissecção da conjuntiva e alguns glaucomas congênitos cujos procedimentos convencionais falharam. E também como alternativa a outros procedimentos ciclodestru$\operatorname{tivos}^{(5,8-11)}$.

As taxas de sucesso de implante para glaucoma relatadas na literatura variam de 57 a $100 \%$, estando relacionadas com doença de base, tempo de seguimento, tipo de implante e critério de sucesso adotado ${ }^{(1)}$. Anteriormente ao uso dos implantes, os resultados em glaucomas refratários não eram considerados aceitáveis, como naqueles glaucomas secundários nos quais as cirurgias fistulizantes raramente alcançavam sucesso devido à proliferação de tecido fibroso com consequente fechamento da fístula ${ }^{(3,8)}$.

As complicações pós-operatórias mais frequentes são principalmente devido à redução excessiva da pressão intraocular, entre elas câmara anterior rasa, atalamia e descolamento de coróide; erosão do trajeto do tubo; obstrução do óstio do tubo; alterações corneais; extrusão do implante; alteração da mobilidade ocular e endoftalmite ${ }^{(1)}$. Em um estudo realizado na Ásia foram avaliadas complicações precoces e tardias. Sendo que as complicações precoces mais encontradas foram hiperemia, descolamento de coróide, uveíte fibrinosa, hemorragia vítrea e endoftalmite. E como tardias, as mais incidentes foram ceratopatia bolhosa, extrusão do tubo, bloqueio do tubo, síndrome pseudo-brown e catarata ${ }^{(9)}$. Taxas de complicações nos implantes de tubo são mais altas do que as ocorridas na trabeculectomia ${ }^{(5)}$.
Considerando a importância do uso de implantes de drenagem para melhor controle no tratamento de glaucomas refratários, este estudo teve como objetivo avaliar aspectos pré e pós-operatórios em pacientes submetidos a esse procedimento, como acuidade visual e pressão intraocular.

\section{MÉTODOS}

Foi realizado um estudo retrospectivo, com revisão de prontuários de todos os pacientes atendidos no setor de glaucoma do Hospital Oftalmológico de Sorocaba (Sorocaba, SP) e submetidos ao procedimento cirúrgico de implante de tubo, no período de janeiro de 2004 a dezembro de 2006.

No estudo foram avaliadas as seguintes variáveis: sexo, idade, olho operado, tipo de glaucoma, número e tipo de cirurgias oculares anteriores, pressão intraocular prévia, após 2 meses, 6 meses, 1 e 2 anos, conforme tempo de seguimento. Também foram verificadas a acuidade visual pré e pós-implante, o número de medicações antiglaucomatosas prescritas pós-implante, os tipos de tubos utilizados e as principais complicações pós-cirúrgicas. A acuidade visual, pressão intraocular prévia e o número de medicações antiglaucomatosas usadas antes da cirurgia foram consideradas como aquelas observadas na última consulta antes da cirurgia. A acuidade visual pós-implante foi obtida a partir da última consulta do seguimento. A pressão intraocular foi medida pelo tonômetro de aplanação Goldmann.

Não foram incluídos no estudo pacientes com acuidade visual sem percepção luminosa pré e pós-operatório e tubos implantados via pars plana.

Foi avaliado um olho por paciente, sendo que pacientes submetidos a cirurgia nos dois olhos, apenas o primeiro olho foi analisado. O tempo de seguimento dos pacientes foi até 2 anos a partir do primeiro dia pós-operatório e com mínimo de 6 meses.

A cirurgia foi considerada sucesso quando a pressão intraocular se manteve entre 6 e $21 \mathrm{mmHg}$ com ou sem medicação hipotensora, independente da acuidade visual.

Para análise estatística, foi utilizado o programa SPSS ${ }^{\circledR}$. As variáveis foram expressas em medidas de tendência central (médias) e desvios-padrão. Foi usado teste do chi-quadrado em tabelas de contigência e incidência e prevalência na estatística epidemiológica.

\section{RESULTADOS}

De todos os pacientes submetidos a cirurgia de implantes de drenagem no período avaliado somente os pacientes com acuidade visual sem percepção luminosa pré e pós-operatório e tubos implantados via pars plana não foram analisados.

Dos 92 pacientes examinados, 3 necessitaram de novo implante de tubo. No total foram avaliadas 95 cirurgias de implantes de drenagem. 
Entre os 93 pacientes a proporção foi de $60(65 \%)$ do sexo masculino e $32(34,7 \%)$ do feminino. A idade variou de 6 a 83 anos, com média de 51,2 $( \pm 18,9)$ anos (Tabela 1).

Dos olhos implantados, $49(51,6 \%)$ olho direito e 46 $(48,4 \%)$ olho esquerdo.

Os tipos de glaucoma tiveram a seguinte distribuição (Tabela 2): 37 olhos $(38,9 \%)$ secundário a transplante de córnea; $24(25,3 \%)$ glaucoma neovascular; $9(9,5 \%)$ póscirurgia de catarata com ou sem implante de lente intraocular, sendo que $1(1,1 \%)$ tinha associado glaucoma de ângulo aberto e cirurgia de catarata; $8(8,4 \%)$ secundário a trauma ocular; $2(2,1 \%)$ sofreram trauma ocular e foram submetidos a transplante de córnea. Seguindo, 5 (5,3\%) glaucoma primário de ângulo aberto; $4(4,2 \%)$ glaucoma de ângulo fechado; 2 $(2,1 \%)$ pós-cirurgia de retina (vitrectomia); $2(2,1 \%)$ glaucoma congênito e $1(1,1 \%)$ secundário a uveíte.

Os tipos de implantes não valvulados utilizados foram: Molteno (78,9\%), Susanna (15,8\%), e entre os valvulados: Ahmed $(3,2 \%)$ e Schoket $(2,1 \%)$.

Não houve diferença significante entre homens e mulheres em relação aos principais tipos de glaucoma $(p>0,001)$. Houve diferença significante quando se correlacionou a indicação de

\begin{tabular}{|c|c|}
\hline \multicolumn{2}{|l|}{ Idade (anos) } \\
\hline Média ( \pm desvio padrão) & $51,2 \pm 18,9$ \\
\hline \multicolumn{2}{|l|}{ Faixa etária, n (\%) } \\
\hline 0 a 10 & $2 \quad(2,1)$ \\
\hline 11 a 20 & $3 \quad(3,2)$ \\
\hline 21 a 40 & $27(28,4)$ \\
\hline 41 a 60 & $26(27,4)$ \\
\hline Acima de 60 & $37(38,9)$ \\
\hline \multicolumn{2}{|l|}{ Sexo, n (\%) } \\
\hline Masculino & $60(65,3)$ \\
\hline Feminino & $32(34,7)$ \\
\hline
\end{tabular}

\begin{tabular}{|lcr|}
\hline \multicolumn{2}{|c|}{$\begin{array}{c}\text { Tabela 2. Frequência segundo o olho e o tipo de glaucoma dos } \\
\text { pacientes submetidos a implante valvular }\end{array}$} \\
Variáveis & $\mathbf{n}$ & $\%$ \\
Olho & & \\
Direito & 49 & 51,6 \\
Esquerdo & 46 & 48,4 \\
Tipo de glaucoma & & \\
Secundário TX & 37 & 38,9 \\
Neovascular & 24 & 25,3 \\
Secundário trauma & 8 & 8,4 \\
GPAA & 5 & 5,3 \\
GPAF & 4 & 4,2 \\
Congênito & 2 & 2,1 \\
Pós-cirurgia catarata & 9 & 9,5 \\
Secundário uveíte & 1 & 1,1 \\
Pós-cirurgia de retina & 2 & 2,1 \\
Secundário TX e trauma & 2 & 2,1 \\
GPAA e pós-cirurgia catarata & 1 & 1,1 \\
TX= Transplante de córnea; GPAA= Glaucoma primário de ângulo aberto; \\
GPAE= glaucoma primário de ângulo fechado. \\
\hline
\end{tabular}

implante de tubo com a faixa etária $(\mathrm{p}<0,05)$. Implantes valvulares foram mais frequentes em pacientes acima de 60 anos.

A acuidade visual pré-implante, avaliada na última consulta antes da cirurgia, foi em sua maioria movimento de mãos $(35,8 \%)$ e conta dedos $(36,8 \%)$, seguido de percepção luminosa $(10,5 \%)$. Quanto à acuidade visual pós-implante, verificada na última consulta do seguimento, não foi observada alteração quanto à ocorrência: movimento de mãos $(32,6 \%)$; conta dedos $(25,3 \%)$ e percepção luminosa $(15,8 \%)$. Não houve diferença significativa da acuidade visual antes e após a cirurgia.

Dentre as 95 cirurgias realizadas, $33(34,7 \%)$ já tinham sido submetidos a duas cirurgias prévias, como facectomia com ou sem implante de lente intraocular, transplante de córnea, trabeculectomia, implante de tubo anterior e/ou vitrectomia. Vinte e cinco $(24,2 \%)$ foram submetidos a uma cirurgia prévia, 8 $(8,4 \%)$ a três e $3(3,2 \%)$ a cinco procedimentos cirúrgicos.

A média da pressão intraocular pré-implante e períodos de 2 meses, 6 meses, 1 ano e 2 anos com uso de medicação e número de paciente sem uso de drogas antiglaucomatosas pode ser observado no quadro 1 . Houve diferença significante entre a pressão intraocular pré-implante e pós dois meses de implante, 1 ano e 2 anos $(p<0,05)$. Não houve significância entre pressão intraocular pré e pós 6 meses, apesar das médias terem sido inferiores em todos os períodos.

Ocorreu perda de seguimento de dois pacientes após um ano de cirurgia, sendo assim, considerada a evolução até esse período.

Avaliando isoladamente a evolução de pacientes submetidos à cirurgia de implante de drenagem por glaucoma pós transplante de córnea (Gráfico 1) e glaucoma neovascular (Gráfico 2) não houve significância entre pressão intraocular pré e pós implante, apesar das médias terem sido inferiores em todos os períodos.

Apesar da conduta terapêutica adotada, em $13(13,4 \%)$ casos não foi observada redução da pressão intraocular durante o seguimento, não atingindo o critério de sucesso estabelecido.

Dos 95 olhos examinados, 38 (40,0\%) não apresentaram complicações pós-cirúrgicas.

As complicações mais frequentes foram: extrusão do implante $(8,5 \%)$, atalamia $(5,4 \%)$, obstrução $(4,2 \%)$, deslocamento do tubo $(3,2 \%)$, Seidel $(2,1 \%)$ e phthisis bulbi $(2,1 \%)$. Sendo que um caso de atalamia evoluiu para phthisis bulbi e outro apresentou extrusão do tubo (Tabela 3).

O tempo médio de seguimento foi de $23( \pm 9,55)$ meses.

\section{DISCUSS ÃO}

Com relação à acuidade visual, não houve diferença significante entre a acuidade visual pré e pós-implante, uma vez que os pacientes apresentavam baixa acuidade visual antes do implante, com prognóstico reservado. Os fatores responsáveis pela baixa acuidade visual foram: glaucoma avançado em pacientes refratários a outros tratamentos, clínico e/ou cirúrgico; evolução natural do glaucoma, por exemplo, o neovascular; a ocorrência de catarata, mais frequente na faixa etária acima de 60 anos; associ- 


\begin{tabular}{|c|c|c|c|c|}
\hline \multicolumn{5}{|c|}{ Pressão intraocular (mmHg) - Média ( \pm desvio padrão) } \\
\hline Pré & Pós 2 meses & Pós 6 meses & Pós 1 ano & Pós 2 anos \\
\hline $32( \pm 11,20)$ & $18( \pm 8,16)$ & $15( \pm 11,00)$ & $16( \pm 11,64)$ & $20( \pm 10,31)$ \\
\hline \multicolumn{5}{|c|}{ Sem uso de medicação - № de pacientes (\%) } \\
\hline Pré & Pós 2 meses & Pós 6 meses & Pós 1 ano & Pós 2 anos \\
\hline $1(1,1)$ & $28(29,5)$ & $39(41,1)$ & $16(30,7)$ & $3(15,0)$ \\
\hline
\end{tabular}

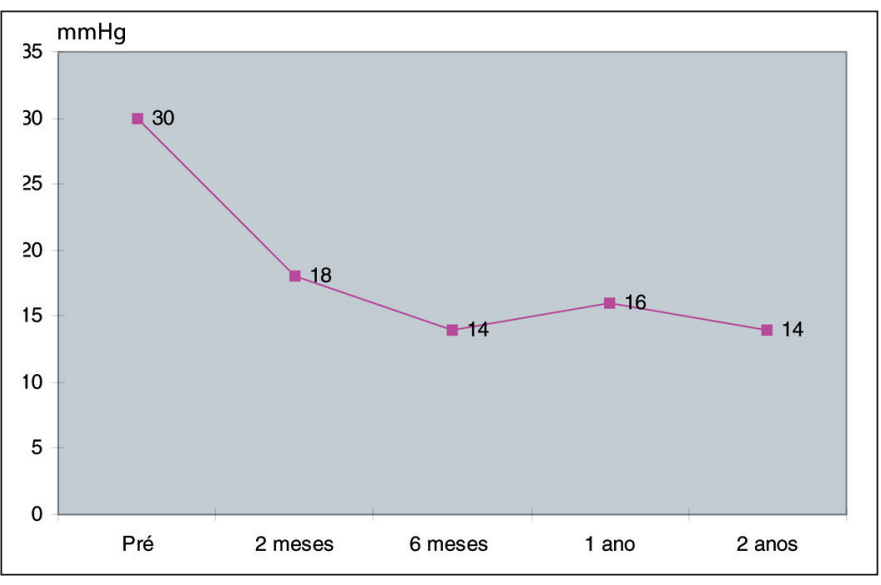

Gráfico1 - Distribuicão da média da pressão intraocular no seguimento pré e pós-operatório com medicação antiglaucomatosa em pacientes submetidos a implante valvular por glaucoma pós transplante de córnea

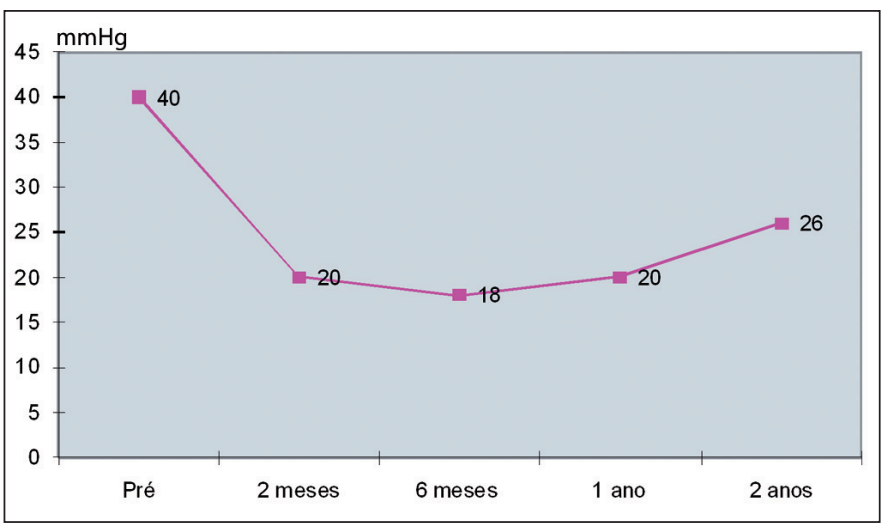

Gráfico 2 - Distribuicão da média da pressão intraocular no seguimento pré e pós-operatório com medicação antiglaucomatosa em pacientes submetidos a implante de drenagem por glaucoma neovascular no Hospital Oftalmológico de Sorocaba no período de janeiro de 2004 a dezembro de 2006. Sorocaba, São Paulo, 2009

ação com outras alterações como hemorragia vítrea em diabéticos com glaucoma neovascular, entre outros.

Neste estudo, a evolução para perda da percepção luminosa $(8,2 \%)$ é concordante com o descrito por Ayyala et al. ${ }^{(12)}$, quando observaram perda de acuidade visual em 5,9\% dos olhos.

Na literatura, Mills et al. ${ }^{(7)}$ relataram que 59\% dos olhos não tiveram melhora da acuidade visual pós-cirurgia. Relataram redução significativa entre os períodos pré e pós-operatório nos

\begin{tabular}{|lcr|}
\hline \multicolumn{2}{|c|}{$\begin{array}{c}\text { Tabela } \\
\text { pacientes submetidos a implante valvular }\end{array}$} \\
Complicação & $\mathbf{n}$ & $\%$ \\
Extrusão & 8 & 8,5 \\
Obstrução & 4 & 4,1 \\
Seidel & 2 & 2,1 \\
Deslocamento & 3 & 3,1 \\
Atalamia & 3 & 3,1 \\
Phthisis bulbi & 1 & 1,0 \\
Atalamia e extrusão & 1 & 1,0 \\
Atalamia e phthisis bulbi & 1 & 1,0
\end{tabular}

pacientes não portadores de glaucoma neovascular, mas todos com glaucoma neovascular perderam a percepção luminosa.

A frequência dos tipos de glaucoma submetidos ao implante de drenagem no estudo foi semelhante a de outros estudos como Higa et al. ${ }^{(1)}$, Almeida et al.$^{(8)}$ e Aung et al..$^{(9)}$, nos quais a principal causa foi glaucoma neovascular e glaucoma pós-cirúrgico. Neste estudo implante de drenagem foi indicado em maior frequência (38,9\%) no glaucoma pós-transplante, uma vez que o estudo foi realizado em hospital de referência para transplante de córnea.

O controle da pressão intraocular sofreu variações em diversos estudos conforme os critérios de sucesso, variando de $67 \%$ a $94 \%$, independente do uso ou não de medicações antiglaucomatosa ${ }^{(4,9,10,13-14)}$. Nesta investigação, essas drogas foram usadas independente do tipo de glaucoma e outros antecedentes oculares.

Outra consideração a ser feita é a redução da pressão intraocular quando comparada com outros métodos clínico e cirúrgico de tratamento, como a trabeculectomia com alto índice de sucesso em casos não complicados. Os implantes de drenagem são muitas vezes realizados em casos graves e refratários e em condições pré-existentes de ruim prognóstico ${ }^{(4)}$.

Neste estudo foi encontrada redução estatisticamente significante da pressão intraocular em 2 meses, 1 ano e 2 anos $(\mathrm{p}<0,05)$, concordando com o estudo desenvolvido por Higa et al. ${ }^{(1)}$. No período de 6 meses houve redução da pressão intraocular, mas esta não foi estatisticamente significante. Isto poderia estar relacionado com o número de medicações usados no pós-operatório, pois $41,1 \%$ dos pacientes não usavam drogas antiglaucomatosas nos primeiros 6 meses.

$\mathrm{Na}$ avaliação da evolução da pressão intraocular em pacientes com glaucoma neovascular ou após transplante de córnea não apresentaram significante diminuição das pressões intraoculares apesar da tendência de redução dos valores du- 
rante o período de seguimento, sendo necessário um maior número de pacientes de cada uma dessas causas de glaucomas refratários para uma melhor avaliação. Faltam na literatura estudos de acompanhamento de cirurgias de implante de drenagem para cada causa específica de glaucoma.

Quanto ao número de medicações usadas, também houve redução entre os períodos pré e pós-operatório nos 2, 6, 12 e 24 meses, apesar de não terem sido estatisticamente significante.

Não ocorreu redução da pressão intraocular em 13,4\%, considerados como sem sucesso, provavelmente por um contínuo remodelamento da cápsula fibrosa com consequente afinamento, perda da permeabilidade, diminuição da drenagem aquosa e falha na formação de uma bolha satisfatória, concordante com os achados de Aung \& $\operatorname{Seah}^{(9)}$.

A frequência de complicações $(40,0 \%)$ foram semelhantes às encontradas em diversos estudos na literatura ${ }^{(1,9,15-17)}$. Essas complicações foram em parte devido à gravidade do glaucoma e ao pobre prognóstico.

As complicações relacionadas ao tubo foram observadas em $16,5 \%$ dos olhos. Na pesquisa realizada por Aung \& $\operatorname{Seah}^{(9)}$ foram verificadas em $12 \%$ dos olhos.

As complicações tiveram ocorrência e evolução independente do tipo de glaucoma, número de cirurgias prévias, idade e sexo. Vale ressaltar que no presente estudo, não foram observadas complicações como descolamento de coróide e retina, endoftalmite e alterações da motilidade ocular.

As correlações com outros estudos são difíceis pela falta de uniformidade de critérios de sucesso, tipos de glaucoma, tempo de seguimento variáveis e diferentes tipos de implante.

Apesar de ser um estudo retrospectivo com levantamento de prontuários, ocorrendo perda de seguimento em dois casos após 6 meses, a pesquisa mostrou resultado efetivo e promissor do implante em diversas causas de glaucoma, variando de acordo com os valores encontrados na literatura.

Implantes de drenagem vêm sendo realizados em glaucomas refratários com alta taxa de sucesso com relação à redução da pressão intraocular, apesar de não ser encontrada uma melhora significativa da acuidade visual. Têm tido cada vez mais aceitação como um bom procedimento para controle do glaucoma e, com isso incentivo para desenvolvimento de novos implantes.

Faz-se necessário a realização de estudos prospectivos com maior número de casos e maior tempo de seguimento.

\section{CONCLUSÃO}

Avaliando os aspectos pré e pós-operatórios observa-se redução da pressão intraocular após os implantes de drenagem, independente do número de medicações antes e após o procedimento. Não ocorrendo melhora em relação à acuidade visual.

\section{ABSTRACT}

Purpose: To evaluate aspects of patients submitted to drainage implant procedures before and after surgery. Methods:
A retrospective study was done, with patients submitted to drainage implant procedures in the Glaucoma Sector of the Hospital Oftalmológico de Sorocaba, from January, 2004 to December, 2006. The following characteristics were evaluated: gender, age, treated eye, glaucoma type, number of previous ophthalmologic surgeries, previous intraocular pressure and check-ups. The visual acuity was observed before and after the implant, the number of glaucoma medications given after the implant, the main complications and the necessity of a second intervention. Results: Among the 92 patients, $65.3 \%(n=60)$ were male and $34.7 \%(n=32)$ were female. The main types of glaucoma were $38.9 \%(n=37)$ due to cornea transplant and $25.3 \%(\mathrm{n}=24)$ to neovascular glaucoma. The intraocular pressure average before implant was $32( \pm 11.29) \mathrm{mmHg}$ and after implant it was $18( \pm 8.11) \mathrm{mmHg}$ in $100 \%(\mathrm{n}=95)$ eyes, $15( \pm 10.89) \mathrm{mmHg}$ in $100 \%(\mathrm{n}=95), 16$ $( \pm 11.64) \mathrm{mmHg}$ in $55.7 \%(\mathrm{n}=54)$ and $20( \pm 10.31) \mathrm{mmHg}$ in $21.6 \%(\mathrm{n}=21)$ for the period of 2 months, 6 months, 1 and 2 years, respectively. The most frequent complications were implant migration $(8.5 \%)$ and shallow anterior chamber $(5.4 \%)$. Conclusion: A satisfactory evolution in the patients after implants, taking into consideration several causes of glaucoma. A significant reduction of intraocular pressure was found in the period of 2 months, at 1 and 2 years $(p<0.05)$. The complications occurred and progressed independent of the type of glaucoma, number of previous surgeries, age or gender.

Keywords: Glaucoma/surgery; Glaucoma drainage implants; Intraocular pressure; Intraoperative complications; Visual acuity

\section{REFERÊNCIAS}

1. Higa FS, Melo Jr LAS, Tavares IM, Tito IR, Mello PAA. Resultados do implante de Susanna em glaucoma refratário. Rev Bras Oftalmol. 2004;63(4):223-9.

2. Heijl A, Leske MC, Bengtsson B, Hyman L, Bengtsson B, Hussein M, Early Manifest Glaucoma Trial Group. Reduction of intraocular pressure and glaucoma progression: results from the Early Manifest Glaucoma Trial. Arch Ophthalmol. 2002;120(10):1268-79. Comment in: Arch Ophthalmol. 2002; 120(10):1371-2. JAMA. 2002;288(20):2607-8. Optom Vis Sci. 2002;79(12):741-2.

3. Omi CA. Implantes valvulares para glaucoma. Arq Bras Oftalmol. 1996;59(1):94-5.

4. Omi CA. Técnica de Shocket modificada no tratamento de glaucomas refratários. (Tese). São Paulo: Universidade Federal de São Paulo. Escola Paulista de Medicina; 1992.

5. Kanski JJ. Oftalmologia clínica: uma abordagem sistemática. $5^{\mathfrak{a}}$ ed. Rio de Janeiro: Elsevier; 2004. p.192-270.

6. Prata JA Jr, Mérmoud A, LaBree L, Minckler DS. In vitro and in vivo flow characteristics of glaucoma drainage implants. Ophthalmology. 1995;102(6): 894-904.

7. Prata JA Jr, Santos RC, LaBree L, Minckler DS. Surface area of glaucoma implants and perfusion flow rates in rabbit eyes. J Glaucoma. 1995:4(4):274-80.

8. Almeida GV, Omi CA, Mandia Júnior C, Cohen R, Kwitko S. Experiência inicial com o implante de Molteno em glaucomas refratários. Arq Bras Oftalmol. 1990;53(3):101-4.

9. Aung T, Seah SK. Glaucoma drainage implants in Asian eyes. Ophthalmology. 1998;105(11):2117-22.

10. Nguyen QH, Budenz DL, Parrish RK $2^{\text {nd }}$. Complications of Baerveldt glaucoma drainage implants. Arch Ophthalmol. 1998;116(5):571-5.

11. Oliveira NM, Oliveira HM, Puzzi L. Avaliação clínica de 17 casos de glau- 
coma tratados com implante de Molteno de placa única. Arq Bras Oftalmol. 1991;54(5):217-20.

12. Ayyala RS, Zurakowski D, Smith JA, Monshizadeh R, Netland PA, Richards DW, et al. A clinical study of the Ahmed glaucoma valve implant in advanced glaucoma. Ophthalmology. 1998;105(10):1968-76.

13. Mills RP, Reynolds A, Emond MJ, Barlow WE, Leen MM. Long-term survival of Molteno glaucoma drainage devices. Ophthalmology. 1996;103(2):299-305. Comment in: Ophthalmology. 1998;105(11):1977.

14. Heuer DK, Lloyd MA, Abrams DA, Baerveldt G, Minckler DS, Lee MB, et al. Which is better? One or two? A randomized clinical trial of single-plate versus double-plate Molteno implantation for glaucomas in aphakia and pseudophakia. Ophthalmology. 1992;99(10):1512-9.

15. Melamed S, Fiore PM. Molteno implant surgery in refractory glaucoma. Surv Ophthalmol. 1990;34(6):441-8. Review.

16. Lloyd MA, Baerveldt G, Heuer DK, Minckler DS, Martone JF. Initial clinical experience with the Baerveldt implant in complicated glaucomas. Ophthalmology. 1994;101(4):640-50.

17. Hodkin MJ, Goldblatt WS, Burgoyne CF, Ball SF, Insler MS. Early clinical experience with the Baerveldt implant in complicated glaucomas. Am J Ophthalmol. 1995;120(1):32-40. 